\title{
Iniciativas Estratégicas No-Tradicionales en las relaciones chino-chilenas: ¿un caso de poder blando de China en América del Sur?
}

\author{
Non-Traditional Strategic Initiatives in \\ Chinese-Chilean relations: a case of soft \\ power of China in South America?
}

Andrés Bórquez*

\section{RESUMEN}

El 2016, Chile fue la sexta nación de América del Sur en elevar sus lazos bilaterales con China al estatus de socio estratégico integral (los otros cinco países son Brasil, Argentina, Venezuela, Perú y Ecuador). Para el caso de China, estas asociaciones conllevan el desarrollo de Iniciativas Estratégicas no Tradicionales (IENs), las cuales van más allá de los parámetros habituales de cooperación en seguridad material y militar. Las IENs surgen como formas de cooperación estratégica basadas en la creación de centros regionales que buscan resolver problemas comunes, incorporando tecnología sofisticada y lógicas de complementariedad estratégica. A pesar de que no existe un límite absoluto entre las iniciativas estratégicas tradicionales y no tradicionales, puesto que no son excluyentes entre sí, estas últimas representan el nuevo marco de poder blando que China intenta establecer en América del Sur, basado en un rol facilitador. En este contexto, Chile ha sido uno de los países que ha acogido la mayor cantidad de IENs

Doctor en Ciencias Políticas y Políticas Internacionales por la Universidad de Fudan, China. Coordinador del Programa de Estudios Chinos del Instituto de Estudios Internacionales de la Universidad de Chile. andres.borquez@uchile.cl. Recibido el 2 de abril de 2019. Aceptado el 15 de octubre de 2019. 
en la región, encontrándose entre las principales el Centro de Astronomía de América del Sur, la plataforma regional de la moneda china y el Centro Regional de Institutos Confucio para América Latina. Este artículo explora las dinámicas detrás de estas iniciativas a través del estudio de caso de las recientes relaciones entre China y Chile.

Palabras clave: Asociaciones estratégicas - Política exterior china - Poder blando - Plataformas regionales.

\section{ABSTRACT}

In 2016, Chile was the sixth nation in South America to raise its bilateral ties with China to the status of integral strategic partners (the other five countries are Brazil, Argentina, Venezuela, Peru and Ecuador). In the case of China, these associations involve the development of Non-Traditional Strategic Initiatives (IENs) which go beyond the usual parameters of cooperation in material and military security. IENs arise as forms of strategic cooperation based on the creation of regional centers that seek to solve common problems, incorporating sophisticated technology and strategic complementarity logic. Although, there is no absolute limit between traditional and non-traditional strategic initiatives, as they're not mutually exclusive. The latter represent the new soft power framework that China tries to establish in South America based on a facilitating role. Understood that, Chile has been one of the countries that has received the largest amount of IENs in the region, amongst them the Astronomy Center of South America, the regional platform of the Chinese currency and the Regional Center of Confucius Institutes for Latin America. This article explores the dynamics behind these initiatives through the case study of the recent relations between China and Chile.

Keywords: Strategic partnerships - Chinese Foreign Policy - Soft Power - Regional Platforms. 


\section{INTRODUCCIÓN}

La cara de las cooperaciones chinas a nivel internacional se encuentra cambiando (Yue 2008; Xun 2017). Junto con sus socios estratégicos, la nación oriental ha desplegado una serie de iniciativas que van más allá de los intereses transaccionales, relacionadas con la complementariedad económica y la generación de plataformas regionales que posicionan la imagen de China como un socio facilitador y sofisticado. Esto se ha observado para el caso latinoamericano donde, por ejemplo, la República Popular China (RPCh) ha establecido una plataforma regional para la expansión de la moneda china, la apertura de centros de investigación en ultramar, la creación de centro de datos, el desarrollo de zonas económicas especiales, la apertura de sedes regionales de empresas estratégicas, etc. (Bórquez, 2019).

El siguiente artículo explora esta dinámica, argumentando que durante los últimos cinco años China ha ido diversificando su forma de cooperar en América del Sur. Beijing ha pasado de la concentración de sus operaciones en la extracción de recursos naturales hacia una cooperación multidimensional, que incluye el desarrollo de iniciativas estratégicas no tradicionales (IENs) con sus socios más cercanos.

Esta lógica envuelve nuevas implicaciones. Se observa cómo China ha ido incorporando una forma de interdependencia compleja, basada en el desarrollo de iniciativas benignas, las cuales en cierta medida compiten con el rol de los países desarrollados. En este sentido, China se ha ido posicionando como una nación facilitadora, donde las iniciativas estratégicas no-tradicionales operan como un elemento de poder blando. En este contexto, el gobierno chino invocó por primera vez el concepto de poder blando en el 17o Congreso del Partido en 2007. El otrora presidente $\mathrm{Hu}$ Jintao asoció este concepto con la creatividad cultural de China. En este sentido, desde un comienzo, las autoridades chinas tomaron distancia de la visión del poder blando estadounidense, poniendo énfasis en elevar la aspiración de la gente al progreso y relacionando el poder blando con la capacidad del pueblo chino para desarrollar creativamente soluciones tangibles (Ping \& Li, 2015). En la actualidad, esto se traduce en una serie de plataformas y mecanismos internacionales centrados en el desarrollo tecnológico, de infraestructura y de conectividad, y que estos elementos sean la fuerzas para potenciar el poder blando de China.

Para explorar esta dinámica, el siguiente artículo se ha estructurado en cuatro partes: primero se realiza una reflexión teórica en torno al rol del poder blando como un juego de suma no nula. En segundo lugar, se discute cómo las iniciativas estratégicas no tradicionales funcionan como un poder blando con características chinas. En 
tercer lugar, se presenta a Chile como un caso crucial del desarrollo de IENs chinas en el cono sur. Finalmente, se discuten posibles implicaciones de estas iniciativas como herramientas de poder blando de China en América del Sur.
Este artículo contribuye preliminarmente con una base teórica y casuística en la discusión sobre si la estrategia de generación de IENs representa un comportamiento con lógicas de suma cero o, por el contrario, incorpora un rol participativo basado en intenciones benignas.

\section{Marco teórico: El PODER blando Como Un JUEGo de SUMA No NULA}

El poder blando o también conocido como la segunda cara del poder es un concepto desarrollado por Nye a principios de los '90, y del cual derivaría su obra publicada en 2004, titulada "El poder blando: los medios para el éxito en la política internacional". Para Nye (2004), el poder no solamente es un acto de coaccionar, sino que también la habilidad de cooptar. Es decir, lograr que otros estados quieran los resultados que desean. En términos prácticos, el poder blando es considerado una herramienta paulatina, donde tanto las naciones como instituciones internacionales disponen de diferentes formas de influencia indirecta, que van desde la persuasión mediante valores culturales hasta la puesta en escena de modelos alternativos de cooperación.

Este artículo reopera el concepto de poder blando para el caso chino como un mecanismo de no suma cero mediante la apertura de iniciativas estratégicas. El desarrollo de actividades a nivel internacional involucra a una empresa compleja donde no solo la distribución de capacidades tiene un impacto, sino también la percepción de que colaborar o competir son elementos necesarios para vivir juntos o para empoderarse. Como menciona el trabajo de Clegg \& Haugaard (2009, 31), las relaciones de poder no siempre implican un juego de suma cero, ya que en algunos casos también involucran interacciones recíprocas, donde ambas naciones comparten los costos y beneficios.

En esta configuración, el poder blando opera como un tipo de cálculo que va más allá de la idea de sobrevivir en un sistema anárquico. Los estados deciden influir ajustando su comportamiento para mostrar la voluntad de estar allí y desbloquear un beneficio mutuo. En este sentido, empoderarse no solo significa maximizar las capacidades y la seguridad, sino que también conlleva la búsqueda de la trascendencia en un mundo lleno de posibles conflictos de intereses. En este punto, el poder blando implica que colaborar y competir no se 
separaren tan fácilmente. Las naciones colaboran para que los agentes puedan competir libremente, al igual que ellos compiten para mantener su soberanía.

Como argumentan Hargreaves-Heap y Varoufakis $(2002,679)$, muchas interacciones entre naciones entrelazan el beneficio mutuo con una porción de conflicto. Para estos autores, el beneficio mutuo es una especie de "equilibrio de equidad" en el que las naciones tienen la necesidad de tener razones para aceptar las convenciones que regulan el comportamiento. Esto, a su vez, genera una búsqueda de equilibrio entre lo que es y lo que debería ser, dando una visión ética a favor de la cooperación, pero reconociendo que existe una fuerza opuesta que es más compleja que el conflicto y que busca mutar.

En dicha configuración, los acuerdos, las redes y las organizaciones son los vehículos que proporcionan el impulso de estas acciones, por lo que están sujetos a influencias e incentivos de intercambio. En esta dinámica, el poder blando funciona como un elemento productivo que rompe con la idea de avanzar mientras limita al otro, pero también implica la capacidad de actuar de manera conjunta y solidaria (Clegg y Haugaard, 2009, 34). En esta misma línea, Nye (2004, 2-4) afirma que influir en terceros implica diferentes caminos, que no solo incluyen coerción y estímulo económico, sino que también hay naciones que tienen características atractivas que son menos fungibles, pero que permiten estimular relaciones de trabajo.

Este comportamiento es una de las explicaciones a la pregunta de por qué la mayoría de los países eligen participar en organizaciones internacionales como la Organización Mundial de Comercio o las Naciones Unidas. Obviamente, no todos los casos operan con estos parámetros, pero estas lógicas se aplican en actividades donde tiene lugar la competencia o colaboración con otros. Este fenómeno se conoce como el efecto del co-actor o coautor en la Teoría de la Facilitación Social (Zajonc, 1965) y el efecto de la internalización en la Teoría de la Influencia Social (Kelman,1958). Extrapolando estas teorías al sistema internacional, la interacción con otras naciones funciona como una fuente de estímulo para mejorar la implementación de algunos compromisos. Estos enfoques apoyan la idea de cooperación bajo el principio de desarrollar modelos de enseñanza y aprendizaje mutuo, donde el énfasis está en el trabajo hacia un objetivo común intercambiando recursos de manera efectiva. Los principales parámetros de estos enfoques son que: a) el país que recibe la influencia puede reconocerla, así como también tiene la posibilidad de negociar las reglas e iniciar procesos de evaluación; b) los países pueden generar iniciativas conjuntas donde la estimulación y la retroalimentación puedan ser sistematizadas, y c) los países aceptan la influencia porque las implicaciones y 
recompensas son satisfactorias. En este contexto, la activación del poder blando aumenta cuando los actores sienten que la contraparte puede evaluar y mejorar el desempeño. Este último elemento responde a una dinámica similar a la mencionada por Nye (2004, 5), donde argumenta que la influencia se basa en la capacidad de dar forma a las preferencias de otros.

En esta misma línea, Wright (2000) y Shiping $(2008,2009)$ enfatizan la interacción de las fuerzas materiales (mundo objetivo) y las ideas (mundo subjetivo) funcionan como la base del poder blando. Wright (2000) argumenta que las relaciones internacionales se están volviendo más complejas, donde el juego no suma cero permite a los gobiernos trabajar juntos para resolver problemas comunes. El autor sostiene que el comportamiento de ganar-ganar es un tipo de fuerza que ha sido crucial para la evolución de las sociedades. Wright afirma que, en el largo plazo, una cooperación basada en un juego no suma cero produce más casos positivos que negativos. Por lo tanto, la cooperación tiende a implicar más beneficios mutuos que el parasitismo. Esta visión confronta la idea de que las naciones colaboran solo como una reacción necesaria a la escasez, dado que los países también tienen impulsos cooperativos basados en la construcción de un sistema internacional benigno y la contribución consciente hacia la interdependencia global.

En la misma línea, Shiping (2008) desarrolla un enfoque social evolutivo centrado en el comportamiento de los estados, que tienen una relación de interdependencia entre sus fuerzas materiales e ideológicas. Estas fuerzas se influyen mutuamente en la construcción de una política exterior, así es como, por ejemplo, una estrategia de seguridad interactúa entre fundamentos materiales (entorno geográfico, naturaleza del sistema internacional, estructura de poder, etc.) y fundamentos de ideas (ideologías, cultura, normas, etc.). Este enfoque rechaza las prácticas de búsqueda de explicaciones monocausales para comprender las interacciones de los estados, poniendo énfasis en la complejidad para establecer relaciones e influencias estratégicas.

Este artículo considera estos enfoques del concepto de poder blando como una solución de no suma nula, donde una nación genera un modelo de expansión de influencia internacional entre una potencia emergente y pequeñas economías pragmáticas, mediante la implementación de iniciativas estratégicas no tradicionales. 


\section{INICIATIVAS ESTRATÉGICAS NO TRADICIONALES Y EL PODER BLANDO CON CARACTERÍSTICAS CHINAS}

Las iniciativas estratégicas se miden bajo los parámetros habituales de cooperación en seguridad a nivel bilateral y multilateral, las cuales incluyen principalmente el intercambio de información, establecimiento de plataformas de suministros de recursos naturales y cooperación en tecnología militar (Schoenshek 1988; Zhang \& Zheng, 2012; Baviera 2016). Sin embargo, durante las últimas décadas han aparecido formas de cooperación estratégicas no tradicionales, que han ido aumentando su importancia en una gobernanza global más dinámica e interconectada. Estas iniciativas tienen entre sus principales características que: i) funcionan como un centro en el que dos países acuerdan establecer una plataforma con implicaciones regionales (por ejemplo, el centro de divisas regional de la moneda china o a la generación de un puerto multimodal); ii) tienen un alto nivel de sofisticación (articulación entre transferencias tecnológicas, desarrollo de una red logística y una gobernanza que incluye participación pública y privada); iii) funcionan bajo la lógica de ganar-ganar en donde ambas naciones incurren en costos y se reparten los beneficios de las IENs.

En América del Sur, China ha focalizado sus IENs en la creación de nodos tecnológicos y de servicios en diferentes áreas como la economía, la cultura y la ciencia. Estas plataformas buscan generar clusters intrarregionales, donde se agrupan instituciones, centros de investigación y empresas del mismo sector para generar soluciones innovadoras a las diferentes necesidades y problemas de una región determinada. En esta configuración, China ha construido un poder blando, agregando sus propias características como herramientas de enfoque sistemático hacia otros países (Bórquez, 2019). Algunos ejemplos de estas iniciativas estratégicas no tradicionales son el puerto multimodal de Chancay en Perú, el centro financiero regional de la moneda china en Chile y la Estación de Espacio Lejano en Neuquén, Argentina.

\section{INICIATIVAS ESTRATÉGICAS NO TRADICIONALES CHINAS: el CASO DE Chile}

A continuación se revisa la evolución de las relaciones sino-chilenas y luego se presentan tres IENs que caracterizan cómo la nación asiática ha ido aumentando su presencia en Chile mediante plataformas tecnológicas, financieras y culturales. 
Relaciones sino-chilenas

El 2019, Chile y China celebran cuarenta y nueve años de relaciones diplomáticas ininterrumpidas, donde el país del sur ha desempeñado un rol pionero en la región. A principios de los años ' 70 , Chile fue el primer país sudamericano en establecer relaciones diplomáticas con la República Popular de China (PRC) y el segundo en América Latina después de Cuba. En la misma línea, en términos de apoyo político, en el año 1999, Chile fue el primer país de América Latina en respaldar el ingreso de la RPC en la Organización Mundial del Comercio y, en 2004, el primer país de la región en reconocer al gigante asiático como economía de mercado. Por otro lado, en términos comerciales, Chile fue el primer país de América Latina en firmar un acuerdo de libre comercio con el gigante asiático. Estos hitos se han convertido en parte fundamental de las relaciones chino-chilenas y, sin duda, han ayudado a consolidar a China como uno de los grandes socios de Chile (Zhang 2006; Gachuz 2012). Otros elementos contemporáneos que han favorecido el fortalecimiento de las relaciones sino-chilenas es que ambos comparten los principios de respeto por la apertura comercial, así como la promoción del multilateralismo y un mundo más multipolar. Ambos países confían en la paz mundial y están convencidos de que la región de Asia Pacífico es uno de los principales polos de desarrollo de la nueva gobernanza global (Errázuriz 2006).

Entre las características más considerables de los lazos chino-chilenos está su trabajo a largo plazo y su evolución gradual para que ambas naciones puedan lograr mejores niveles de desarrollo. En más de cuatro décadas de relaciones diplomáticas, Chile y China han profundizado progresivamente su acercamiento mutuo. El fortalecimiento de los vínculos institucionales y la intensificación de las visitas de alto nivel permitieron la incorporación de nuevas áreas de trabajo (Pérez Le-fort 2006). Esto se ha reflejado en el constante progreso de las vinculaciones entre ambos países, por ejemplo, el 2016 elevaron la relación bilateral a una asociación estratégica integral y se firmaron nuevos memorandos para profundizar el acuerdo de libre comercio que llevaba diez años en vigencia. Este progreso muestra cómo los vínculos entre ambas naciones están diversificando e incorporando campos tan lejanos como el espacio exterior, la biotecnología y el desarrollo de nuevos materiales.

Las autoridades chilenas han optado por una actitud política pragmática hacia Beijing, esto se ha expresado en el respeto mutuo de la soberanía y la integridad territorial, en el apoyo a la "política de una China" y en el otorgamiento del estatus de economía de mercado. Chile considera a China como uno de los socios más importantes en la región de Asia Pacífico. 
Esto se ha reflejado, por ejemplo, en la búsqueda de un diálogo permanente a través de visitas oficiales y la generación de mecanismos de consulta. Durante los últimos 27 años, todos los presidentes chilenos, independientemente de su ideología política, han realizado al menos una visita oficial a China (Heine 2016). Al mismo tiempo, Chile ha firmado diferentes acuerdos de cooperación para institucionalizar la relación entre ambos países (acuerdos de asociación estratégica integral y acuerdos de libre comercio, entre los más importantes).

En términos de asuntos exteriores, Chile comparte la misma idea de buscar un mundo multipolar y un orden económico internacional justo. En este sentido, Chile ha prestado atención a la iniciativa de la Franja y la Ruta y al Área de Libre Comercio de Asia-Pacífico (FTAAP) como iniciativas complementarias en la región de Asia Pacífico (Yu 2018). Por un lado, la iniciativa de la nueva ruta de la seda se visualiza como una oportunidad para reducir las barreras geográficas y comunicacionales a través del desarrollo de mayor conectividad e infraestructura. Por otro lado, el fTAAP está dimensionado como una iniciativa para superar los temores proteccionistas causados por la automarginación estadounidense del acuerdo Transpacífico de Cooperación Económica (TPP en sus siglas en inglés) y para actualizar el nivel de vínculos en el Foro de Cooperación Económica Asia Pacífico (APEC en sus siglas en inglés).
En términos económicos, Chile ha sido uno de los países que más se ha beneficiado de las relaciones comerciales con China.El temprano acuerdo de libre comercio entre las dos naciones ha permitido a Chile tener un acceso prioritario al mercado asiático, además de importar productos de alta tecnología a un precio competitivo. El año pasado, luego de una evaluación positiva del TLC por ambas partes, comenzó una fase de profundización, en la cual Chile busca seguir siendo un proveedor de recursos primarios, pero también atraer nuevas inversiones chinas, especialmente en infraestructura y en el desarrollo tecnológico de la minería y la agricultura. Por otro lado, Chile busca posicionarse como una plataforma comercial para el ingreso de las principales marcas de productos y servicios chinos al mercado sudamericano (Pérez Le-fort 2006).

\section{Centro financiero regional de la moneda china}

En mayo de 2015, en el marco de la visita a Chile del Primer Ministro chino, Li Keqiang, se establecieron acuerdos de cooperación financiera entre los bancos centrales, con el objetivo de facilitar el uso de la moneda china en las transacciones internacionales entre ambas regiones. Esta plataforma se operacionalizó mediante la firma de tres acuerdos: canje de divisas (Currency SWAP Agreement), el acuerdo de compensación bancaria (Renminbi Clearing Bank) y la unión al programa 
de Inversionistas Institucionales Extranjeros Calificados en Renminbi (Renminbi Qualified Foreign Institutional Investor Program, RQFII). Lo anterior permitió establecer en Chile el primer centro financiero de la moneda china en América Latina, posicionado al país como una plataforma financiera para su internacionalización en la región. ${ }^{1}$ Esta iniciativa permite a China incrementar su cooperación financiera e inversión en toda la región utilizando a Chile como puente, puesto que se proceden a efectuar pagos en la moneda china reduciendo costos de operaciones financieras, pagos internacionales y financiamiento del comercio exterior. Cabe señalar que, a pesar del impulso inicial, todavía esta iniciativa no logra posicionarse como una plataforma regional y las empresas locales prefieren concretar sus negocios en otros centros como el de Hong Kong. Estas plataformas son un entramado complejo y, por ende, se necesitan estudios y discusiones entre las partes para que logren su promesa de establecer un centro dinámico y que otorgue ganancias de largo plazo para ambas partes.

\section{Centro Sudamericano para la Astronomía}

El Centro de Astronomía de América del Sur de la Academia China de Ciencias es una plataforma para la colaboración en investigación astronómica y en el desarrollo de tecnologías limpias para América del Sur. Para la ejecución de esta plataforma, Chile y China firmaron en el año 2016 el Acuerdo de Colaboración de Entendimiento entre la Academia China de Ciencias y la Comisión Nacional de Investigación Científica y Tecnológica de Chile. Este marco permitió, en julio de 2018, abrir la primera oficina del centro sudamericano en la región de Antofagasta. Esta oficina es coordinada con la Universidad Católica del Norte con la cual planifican construir en el Cerro Ventarrones el primer observatorio astronómico con tecnología china en Chile. Esto permitirá, al igual que los otros convenios con observatorios internacionales instalados en Chile, asegurar el 10\% del tiempo para el uso exclusivo de investigadores chilenos. ${ }^{2}$ Otra iniciativa complementaria es el primer Data Center para el desarrollo de la astro-informática regional. Este centro de datos se sustenta en el acuerdo de cooperación firmado el 2015 entre la Academia de Ciencias de China, la sucursal chilena de Huawei y la Universidad Técnica

2 Fuente: Chinese Academy of Sciences South America Center for Astronomy http://www.cassaca.org/es/ 
Federico Santa María, y consiste en un centro de datos modular, el cual posee una capacidad de $1 \mathrm{~PB} .{ }^{3}$ Las creaciones de nuevas instancias de cooperación han ido posicionando a Chile, junto con China, como plataforma regional para el desarrollo científico y de las telecomunicaciones. Dada la naturaleza de largo plazo de este tipo de plataformas, una vía factible de concretarlas ha sido la cooperación académica y científica (Bórquez 2019).

\section{Centro Regional del Instituto Confucio para América Latina}

El Centro Regional del Instituto Confucio para América Latina (CRICAL) se estableció con la finalidad de promover y coordinar desde Chile el estudio de la lengua y cultura china a nivel latinoamericano. Entre las principales funciones que tiene esta nueva institución, se encuentra posicionar la oficina de Santiago como plataforma estratégica en los procesos de profundización de las relaciones culturales de la región con la República Popular China. En términos operacionales, este centro es el encargado de organizar tres funciones para los 29 centros Confucio de la región: i) coordinar actividades de capacitación de los profesores y encuentros culturales; ii) publicar materiales educativos y culturales, y iii) centralizar la planificación regional a través de un consejo asesor. ${ }^{4}$ Este centro se materializó bajo la firma de un convenio entre la oficina Central del Idioma Chino Mandarín, también conocida como Hanban, y la Universidad Santo Tomás de Chile, donde se establece que esta última provee espacio dentro de la universidad para realizar sus actividades. Como externalidad positiva, en 2019 la Universidad Santo Tomás se ha posicionado como una de las instituciones regionales líderes en la enseñanza del chino mandarín con la apertura de 19 sedes del Instituto Confucio a nivel nacional.

\section{Discusión}

El aumento de la presencia de China en América del Sur no solamente puede entenderse desde el crecimiento económico, sino también mediante la incorporación de mecanismos e

3 Fuente: Sitio oficial Universidad Federico Santa María http://www.noticias.usm. cl/2015/09/16/usm-inaugura-el-data-center-astroinformatico-mas-grande-del-pais/ iniciativas de cooperación sofisticadas. China está diversificando sus formas de cooperación al tiempo que expande su influencia en nuevas regiones. Este enfoque incluye la capacidad de la nación asiática para persuadir a sus

4 Fuente: página oficial Centro Regional del Instituto Confucio para América Latina https://www.crical.cl/quienes-somos/ 
socios en la generación de iniciativas sofisticadas con un alto nivel de vinculación estratégica. En este contexto, las IENs chinas son instancias para la operacionalización de las asociaciones estratégicas establecidas por China en la región. Esto le ha permitido a la nación asiática ir alcanzando sus objetivos materiales y, al mismo tiempo, la oportunidad de influir en otros países (Bórquez 2019).

A continuación, se discuten tres elementos sobre las IENs que deben ser profundizados en futuros estudios, con el objetivo de evaluar si estos instrumentos son parte del poder blando chino en América del Sur. En primer lugar, debido al poco tiempo de la puesta en marcha estas iniciativas en Chile, aún es muy pronto para saber si estas plataformas tienen el impacto esperado. En este sentido, al revisar algunos casos como, por ejemplo, el centro financiero regional, todavía no queda del todo claro cuáles son los beneficios de largo de largo plazo para los ejecutores chilenos. Sin duda, para los usuarios finales esta plataforma les permite reducir sus costos transaccionales. No obstante, para el sistema financiero chileno esta plataforma funciona como una especie de reemplazo de otras iniciativas ligadas al uso de monedas como el dólar. En este sentido, es pertinente revisar los impactos esperados y, por ejemplo, ver si efectivamente con este centro aumenta la llegada de inversiones chinas en Chile y la región.
En segundo lugar, la nación asiática mediante estas iniciativas de poder blando insta a naciones periféricas a participar en los nuevos procesos de gobernanza global. Esto también se puede observar en otras regiones a través del desarrollo de la Nueva Ruta de la Seda China (Belt and Road Initiative en sus siglas en inglés). A pesar de que la estrategia de cooperación internacional china se basa cada vez más en un liderazgo concertado, aún no queda claro para los países de América del Sur si este nuevo rol será similar a las cooperaciones de países desarrollados o, por defecto, se mantendrá como un tipo de cooperación sur-sur. Este elemento deberá ser estudiado con más detalle en el mediano plazo, principalmente porque las IENs todavía representan una parte minúscula de las relaciones entre ambas regiones. En esta misma línea, cabe señalar que hasta el momento la presencia de China en América del Sur todavía se concentra en recursos naturales y materias primas, lo que ha derivado en una reprimarización de las estructuras productivas de la región, tendencia similar a lo que se venía observando con la llegada de capitales norteamericanos o europeos.

En tercer lugar, se observa que las IENs muestran un alejamiento de la política de bajo perfil heredada por Deng Xiaoping, la cual es modificada por una política exterior más asertiva por parte del liderazgo del actual presidente chino Xi Jinping (Wong 2005, 
Xuetong 2014). Pese a que, en términos de poder blando, la ventaja de Estados Unidos es significativa, China ha comenzado a determinar su propio camino para fortalecer su imagen internacional. En este sentido, a diferencia del poder blando norteamericano, centrado en la generación de valores universales y en exportar dimensiones culturales mediante los medios de comunicación de masas, la nación asiática busca posicionarse como un socio atractivo para solucionar problemas globales. Esto se traduce en la generación de una serie de plataformas y mecanismos de ultramar centrados en el desarrollo tecnológico, de infraestructura y de conectividad. Estas iniciativas han sido principalmente desarrolladas en países en vías de desarrollo que comparten problemas similares con China. En el caso de América del Sur, este rol históricamente ha sido desplegado por Estados Unidos y las instituciones internacionales que lidera. En este contexto, la avanzada de China mediante sus iniciativas mezclada con la prolongación de su guerra comercial con Estados Unidos ha planteado un nuevo escenario geopolítico en la región, el que deberá seguirse con atención en nuevos estudios.

\section{Conclusión}

Las iniciativas estratégicas no tradicionales (IENs) representan un elemento clave del poder blando de China basado en el establecimiento de liderazgo compartido, donde la nación asiática articula entre sus necesidades de seguridad material (por ejemplo, la adquisición de recursos naturales) y su vocación ideacional basada en generar interdependencias complejas (por ejemplo, la apertura de plataformas concertadas con sus socios estratégicos para lograr objetivos comunes, basados en la complementariedad económica). En palabras del propio Presidente chino, la segunda economía mundial busca, más allá de su crecimiento económico y político, mantener la cooperación bajo los parámetros de ganancias y beneficios mutuos como principio normativo (Xi Jinping, n.d.).

En este estudio preliminar, Chile ha sido un caso crucial en el desarrollo de cooperaciones estratégicas no tradicionales en América Latina, destacando la multidimensionalidad de estas iniciativas. China y Chile, a través de la generación de iniciativas sofisticadas, han ido profundizando el nivel de las relaciones, pasando desde el mero intercambio comercial hacia la búsqueda de una cooperación compleja. Estas naciones aprovechan la complementariedad económica y ventajas comparativas de cada país para trabajar estratégicamente hacia un objetivo común. En este sentido, las 
iniciativas estratégicas no tradicionales emergen como una alternativa de cooperación que permite a pequeñas naciones, como Chile, avanzar en el camino hacia el desarrollo. Los países no solo prestan atención a la transacción de productos primarios, sino que también incluyen iniciativas de largo plazo, enfocadas en fortalecer la investigación científica, la transferencia de tecnologías y en incentivar una cultura más propensa a generar valor agregado.

Aunque las IENs establecidas en América del Sur todavía representan un volumen minúsculo de las inversiones y cooperaciones chinas en la región, vale la pena señalar que China ha sabido utilizar estos instrumentos de una manera compleja, extendiendo el horizonte de impacto en las cooperaciones internacionales. La nación asiática ha entendido que las IENs son instrumentos ganar-ganar, donde las economías pequeñas y medianas visualizan estas iniciativas como una alternativa de cooperación sofisticada a las que suelen realizar con las potencias occidentales. En este sentido, uno de los méritos de la política exterior de China ha sido que en poco tiempo ha entendido que el mundo no es una unidad sociopolítica homogénea, por lo cual ha estado articulando dispositivos de cooperación basados en diferentes perfiles de naciones. En esta línea, se observa que los próximos años fortalecerá esta tendencia en América del Sur, ya que China ha establecido estudios de viabilidad para el desarrollo de IENs en países como Perú y Colombia. Esto ha marcado un movimiento en el rompecabezas geopolítico en la región, donde China logra llamar la atención de la gran parte de los países, incluyendo naciones históricamente cercanas a Estados Unidos.

A nivel internacional, esto también se ha visto reflejado, ya que China, a través de la Nueva Ruta de la Seda, ha ido estableciendo IENs con varias naciones de Asia, como Paquistán, Malasia y Sri Lanka. En este contexto, esta tendencia se enmarca en cómo China está construyendo nuevas reglas de intercambio global para adaptarse a la cambiante situación económica y política internacional.

Estos resultados son coherentes con el marco teórico planteado, en el sentido de que las iniciativas estratégicas no tradicionales se posicionan como una herramienta cooperativa de beneficio mutuo basado en la generación de plataformas concertadas y la estimulación mutua para resolver problemas comunes. Esto se observa en la revisión de la constitución de las plataformas chinas en Chile, como lo son la financiera, científica y cultural. Por último, cabe resaltar que este estudio es de carácter preliminar, por lo cual se requieren nuevos análisis y revisión de casos para ir estableciendo un marco teórico para evaluar el comportamiento de las cooperaciones estratégicas no tradicionales. 


\section{Bibliografía}

Baviera, A. (2016). China's Strategic Foreign Initiatives Under Xi Jinping An ASEAN Perspective. China Quarterly of International Strategic Studies 2(1): 57-79.

Bórquez, A. (2019). Exploring the nontraditional foreign investment in Sino-Latin American relations: The Chilean case. Competition and Regulation in Network Industries. https://doi. org/10.1177/1783591719877592

Stewart, C. \& Haugaard, M. (2009). The SAGE handbook of Power. London: SAGE publications Ltd.

Errázuriz, O. (2006). Las relaciones de Chile y China: del simbolismo a la acción. Estudios Internacionales 38 (154): 123-136.

Gachuz, J. (2012). Chile`s Economic and Political Relationship with China. Journal of Current Chinese Affairs 41(1): 133-154.

Hargreaves-Heap, Sh. \& Varoufakis, Y. (2002). Some experimental evidence on the evolution of discrimination, cooperation and perception of fairness. The Economic Journal 112 (July): 679-703.

Heine, J. (2016). The Chile Paradox. Asian Perspective 40 (4): 653-673.

Kelman, H.C. (1958). Compliance, Identification, and Internalization: Three Processes of Attitude Change. The Journal of Conflict Resolution 2 (1):51-60.

Nye, J. (2004). Soft Power: The Means to Success in World Politics. New York: Public Affairs.

Pérez Le-fort, M. (2006). Relaciones sino-chilenas bajo nuevas circunstancias. Estudios Internacionales 152 (38): 123-136.
Ping, X. \& Wang, L. (2015). The China Model vs. American Soft Power: Going Global and Peaceful. Istanbul Gelisim University Journal of Social Sciences 2(2): 154-170.

Shiping, T. (2009).“The Security Dilemma: A Conceptual Analysis". Security Studies, 18:587-623.

Shiping, T. (2008). "From Offensive to Defensive Realism. A social evolutionary interpretation of China's Security Strategy", en Ross, R. \& Feng, Z. (eds.) China's ascent: Power, security, and the future of international politics. New York: Cornell University Press

Schonshek, J. (1988). Confusion and false advertising of the strategic defense initiatives. International Journal on World Peace, 5 (3): 69-107.

Wang, Y. (2009). Transition of China's Diplomacy and Foreign Relations. China \& World Economy, 17(3), 93-101.

Wong, Y-C. (2005). From Deng Xiaoping to Jiang Zemin: Two decades of political reform in the People's Republic of China. Lanham: University Press of America. Wright, R. (2000). Non-zero: the logic of human Destiny. New York: Random House Inc.

Xuetong, Y. (2014). "From Keeping a Low Profile to Striving for Achievement”. The Chinese Journal of International Politics 7 (2): 153-184.

Xun, P. (2017). China's Network Strategy for Seeking Great Power Status. The Chinese Journal of International Politics 10(1): 1-29. 
Yue, J. (2008). "Peaceful Rise of China: Myth or Reality?". International Politics 45(4), 439-456.

Yu, L. (2018). China `s Free Trade Area Strategy: an alternative avenue to China's peaceful rise? The Social Science Journal (article in press).

Zajonc, R. (1965).“Social Facilitation”. Science 149 (3681): 269-274.
Zhang, J. \& Zheng, X. (2012). The Role of Nontraditional Security in China-US Relations: common ground or contradictory arena? Journal of Contemporary China, 21, 623-636.

Zhang, X. (2006). El entendimiento sobre el Tratado de Libre Comercio entre China y Chile. Estudios Internacionales 152 (38): 113-121. 